5. Ghinai I, McPherson TD, Hunter JC, et al. First known person-to-person transmission of severe acute respiratory syndrome coronavirus 2 (SARSCoV-2) in the USA. Lancet 2020;395:1137-1144.

6. Canova V, Schlpfer HL, Piso RJ, et al. Transmission risk of SARS-CoV-2 to healthcare workers-observational results of a primary care hospital contact tracing. Swiss Med Wkly 2020;150.

7. Ng K, Poon BH, Puar THK, et al. COVID-19 and the risk to health care workers: a case report. Ann Intern Med 2020;172:766-767.
8. Wang D, Hu B, Hu C, et al. Clinical characteristics of 138 hospitalized patients with 2019 novel coronavirus-infected pneumonia in Wuhan, China. JAMA 2020;323:1061-1069.

9. Zou L, Ruan F, Huang M, et al. SARS-CoV-2 Viral load in upper respiratory specimens of infected patients. N Engl J Med 2020;382:1177-1179.

10. COVID-19 data for Kent County, Michigan. accessKent website. https:// www.accesskent.com/Health/covid-19-data.htm. Accessed June 11, 2020.

\title{
Universal screening for the SARS-CoV-2 virus on hospital admission in an area with low COVID-19 prevalence
}

\author{
Sangeeta R. Sastry MD, Rachel Pryor RN, MPH, Jillian E. Raybould MD, Julie Reznicek DO, Kaila Cooper RN, MSN, \\ Amie Patrick RN, MSN, Shelley Knowlson RN, Pamela Bailey DO, MPH, Emily Godbout MD, MPH, Michelle Doll MD, MPH, \\ Michael P. Stevens MD, MPH and Gonzalo Bearman MD, MPH \\ Division of Infectious Diseases, Virginia Commonwealth University, Richmond, Virginia
}

Asymptomatic persons contribute to widespread transmission of the severe acute respiratory coronavirus virus 2 (SARS-CoV-2) and the coronavirus disease 2019 (COVID-19) pandemic. ${ }^{1}$ Published reports from areas of high COVID-19 incidence in the United States suggest that a significant percentage of asymptomatic persons are in healthcare systems. In 2 New York City (NYC) hospitals, $13.7 \%$ of asymptomatic pregnant women admitted for delivery tested positive for SARS-CoV-2 virus. ${ }^{2}$ Similarly, the nursing facility in Washington state with the earliest death from COVID-19 infection and the first healthcare worker infected in the United States, reported $>50 \%$ positivity of their asymptomatic residents for the virus. ${ }^{3}$ Universal screening of healthcare populations may prevent in-hospital transmission of SARS-CoV-2 virus. However, testing resources and personal protective equipment (PPE) supplies to effectively isolate positive asymptomatic persons are currently limited, resulting in provider safety concerns. Upon developing real-time reverse-transcriptase polymerase chain reaction (rRT-PCR) tests in-house with $>98 \%$ sensitivity, as well as increasing the availability of PPE at our institution, we initiated universal screening of patients on hospital admission using nasopharyngeal swabs to identify and isolate asymptomatic positive patients to prevent in-hospital transmission of SARS-CoV-2. We report our experience with universal screening of asymptomatic hospitalized persons, including a comparison of demographics between symptomatic and asymptomatic populations.

\section{Methods}

On April 27, 2020, our 1,000-bed academic center instituted universal SARS-CoV-2 testing of patients on hospital admission. Clinicians performed COVID-19 symptom screening using clinical criteria reported in the literature. ${ }^{4}$ They designated patients as symptomatic or asymptomatic when ordering the test. An infectious diseases physician conducted chart review of asymptomatic

Author for correspondence: Sangeeta R. Sastry, E-mail: Sangeeta.Sastry@vcuhealth.org Cite this article: Sastry SR, et al. (2020). Universal screening for the SARS-CoV-2 virus on hospital admission in an area with low COVID-19 prevalence. Infection Control \& Hospital Epidemiology, 41: 1231-1233, https://doi.org/10.1017/ice.2020.358 positive patients to confirm accuracy of classification. Asymptomatic patients were not isolated; test turnaround time was 6-24 hours.

Statistical analyses were performed with the Fischer exact tests and paired $t$ tests to compare asymptomatic and symptomatic positive patients using SAS version 9.4 software (SAS Institute, Cary, NC).

\section{Results}

Between April 27, 2020, and May 18, 2020, when the hospital averaged at $60 \%-70 \%$ capacity, we performed 1,811 SARS-CoV-2 tests on nasopharyngeal specimens: $1,335(74 \%)$ were asymptomatic, $420(23 \%)$ were symptomatic, $56(3 \%)$ were incorrectly ordered. Of the 1,755 tests in this analysis, overall positivity for SARCoV-2 virus was 79 (4.5\%). Of 79 patients, 12 were asymptomatic $(15 \%)$ and 67 were symptomatic (85\%). Of 1,335 asymptomatic patients, 12 tested positive, for a rate of $\sim 1 \%$. Of 420 symptomatic patients, 67 tested positive, for a rate of $16 \%$. No test converted to positive among asymptomatic patients while hospitalized.

A comparative analysis of patients with positive SARS-CoV2 tests is listed in Table 1 . The mean age of asymptomatic patients was 37 years (SD, 19.71) versus a mean age of 59 years (SD, 13.08) among symptomatic patients $(P=.0020)$. Hispanic patients were more likely to be asymptomatic ( 7 of 12$)$ than symptomatic ( 9 of 67$)$ at the time of testing ( $58 \%$ vs $13 \% ; P=.0017)$. We observed no difference in positivity rate on admission of asymptomatic versus symptomatic patients $(P=.21)$. In addition, 5 asymptomatic positive women were pregnant (5 of $12,42 \%$ ); no symptomatic patients were pregnant $(P \leq .0001)$. A baby born to an asymptomatic SARS-CoV-2-positive mother tested positive at 48 hours of life, and 1 asymptomatic, SARS-CoV-2-positive, immunocompromised patient was receiving chemotherapy for breast cancer. One asymptomatic patient developed a fever during hospitalization, and another was readmitted within 14 days of testing positive, both of these events were not considered to be related to COVID-19. 
Table 1. Comparative Analysis Between Asymptomatic and Symptomatic Patients With Positive SARS-CoV-2 Virus Tests

\begin{tabular}{|c|c|c|c|c|}
\hline \multicolumn{2}{|c|}{$\begin{array}{l}\text { Demographic Characteristics of } \\
\text { Asymptomatic Patients, } \\
\text { SARS-CoV-2-Positive Patients } \\
\text { (N=12, } 0.62 \%)\end{array}$} & \multicolumn{2}{|c|}{$\begin{array}{c}\text { Demographic Characteristics of Symptomatic } \\
\text { Patients, } \\
\text { SARS-CoV-2-Positive Patients } \\
(\mathrm{N}=67,3.5 \%)\end{array}$} & \multirow{2}{*}{$\begin{array}{c}P \text { Value } \\
.0020\end{array}$} \\
\hline Mean age, y (range) & $\begin{array}{c}37(0-67) \\
(S D, 19.71)\end{array}$ & Mean age, y (range) & $\begin{array}{l}59(12-78) \\
(S D, 13.08)\end{array}$ & \\
\hline & No. (\%) & & No. $(\%)$ & \\
\hline Race/ethnicity & & Race/ethnicity, y (ra & & \\
\hline Hispanic & $7(58)$ & Hispanic & $9(13)$ & .0017 \\
\hline African American & $4(33)$ & African American & $36(54)$ & .22 \\
\hline Caucasian & $1(8)$ & Caucasian & $14(21)$ & .44 \\
\hline Other & $0(0)$ & Other & $8(12)$ & \\
\hline Gender & & Gender & & \\
\hline Male & $5(42)$ & Male & $42(63)$ & .21 \\
\hline Female & $7(58)$ & Female & $25(37)$ & \\
\hline Pregnant & $5 / 7(42)$ & Pregnant & 0/25 (0) & $\leq .0001$ \\
\hline
\end{tabular}

Note. SD, standard deviation.

\section{Discussion}

Universal screening for the detection of SARS-CoV-2 at our institution revealed that during the study period, the number of asymptomatic persons admitted to the hospital was relatively small. Our health system had a relatively low number of confirmed SARS-CoV-2-positive COVID-19 patients $(\mathrm{n}=82)$ admitted during the observed 3-week interval, compared to 4,000 patients admitted to an NYC hospital reporting the use of convalescent serum for the treatment of COVID-19 in a similar time frame. ${ }^{5}$ Although low prevalence of asymptomatic patients has limited generalizability to areas with higher rates of infection, it is valuable information for patients, healthcare workers, and epidemiology programs in similar areas of COVID-19 prevalence.

During our study period, $7.6 \%$ of all admitted patients were Hispanic and $43.5 \%$ were African American, yet 11 of 12 (91.7\%) asymptomatic patients who screened positive were African American or Hispanic. A similar trend was observed in other studies. ${ }^{6,7}$ Furthermore, a higher proportion of pregnant women have asymptomatic infection, which supports screening of peripartum women. Consistent with the literature, asymptomatic patients were younger than those who presented to our healthcare system with COVID-19 symptoms. ${ }^{8}$

The potential benefits of universal SARS-CoV-2 screening are many and are likely to increase with escalating COVID-19 incidence. In hospitalized patients, detection of asymptomatic infection can guide hospital isolation practices, bed assignments, and the use of PPE. ${ }^{2}$ For healthcare workers, it might improve workforce depletion by unnecessary quarantine, reduce transmission in asymptomatic cases, contain the virus in healthcare settings, and protect hospital staff from infection. In the community, mass testing can identify asymptomatic cases and assist in eliminating the SARS-CoV-2 virus, as reported in a village near Venice, Italy. ${ }^{9}$

However, there are barriers to universal screening. Current testing capacity and test turnaround time, staffing shortages, and availability of healthcare workers skilled to perform nasopharyngeal swabbing currently limit widespread feasibility. Patient discomfort from nasopharyngeal sample collection is another potential barrier to universal screening.

This study has several limitations. The sample size was small, and the study was conducted at a single center. In an area with high prevalence of COVID-19 infection, asymptomatic screening would likely identify more asymptomatic cases. However, sensitivity of a test in asymptomatic persons cannot be precisely defined. We add to the body of literature on SARS-CoV-2 testing of asymptomatic patients at the time of hospital admission. More data on universal screening is necessary to evaluate the clinical impact on healthcare systems and to define optimal screening strategies of high-risk groups for asymptomatic COVID-19 infection.

Acknowledgments. None.

Financial support. No financial support was provided relevant to this article.

Conflicts of interest. All authors report no conflicts of interest relevant to this article.

\section{References}

1. Gandhi M, Yokoe DS, Havlir DV. Asymptomatic transmission, the Achilles' heel of current strategies to control COVID-19. N Engl J Med 2020;382:21582160.

2. Sutton D, Fuchs K, D'Alton M, et al. Universal screening for SARS-CoV-2 in women admitted for delivery. $N$ Engl J Med 2020;382:2163-2164.

3. Arons MM, Hatfield KM, Reddy SC, et al. Presymptomatic SARS-CoV-2 infections and transmission in a skilled nursing facility. $N$ Engl J Med 2020;382:2081-2090.

4. Sun Y, Koh V, Marimuthu K, et al. Epidemiologic and clinical predictors of COVID-19. Clin Infect Dis 2020 [Epub ahead of print]. doi: 10.1093/cid/ ciaa322.

5. Liu STH, Lin HM, Baine I et al. Convalescent plasma treatment of severe COVID-19: a matched controlled study. medRxiv 2020 May 22. doi: 10. $1101 / 2020.05 .20 .20102236$.

6. Garg S, Kim L, Whitaker M, et al. Hospitalization rates and characteristics of patients hospitalized with laboratory-confirmed coronavirus disease 2019COVID-NET, 14 states, March 1-30, 2020. Morb Mortal Wkly Rep 2020;69:458-464. 
7. Kirby T. Evidence mounts on the disproportionate effect of COVID-19 on ethnic minorities. Lancet Respir Med 2020 May 10 [Epub ahead of print]. doi: 10.1016/S2213-2600(20)30228-9.

8. Ma Y, Xu Q, Wang F et al. Characteristics of asymptomatic patients with SARS-CoV-2 infection in Jinan, China. Microbes Infect 2020;22:212-217.
9. Day M. COVID-19: identifying and isolating asymptomatic people helped eliminate virus in Italian village. BMJ 2020;368:m1165. doi: 10.1136/bmj. m1165.

\title{
Utility of asymptomatic inpatient testing for COVID-19 in a low-prevalence setting: A multicenter point-prevalence study
}

\author{
Anthony D. Bai MD ${ }^{1}$, , Xena X. Li MD¹, Mohammed Alsalem MD, Sarah Khan MD², Marek Smieja MD³, Dominik Mertz MD \\ and Zain Chagla MD ${ }^{5}$ \\ ${ }^{1}$ Division of Infectious Diseases, McMaster University, Hamilton, Ontario, Canada, ${ }^{2}$ Infection Prevention Control, McMaster Children's Hospital, Hamilton, Ontario, \\ Canada, ${ }^{3}$ Laboratory Medicine, St Joseph's Healthcare Hamilton and Hamilton Health Sciences, Hamilton, Ontario, Canada, ${ }^{4}$ Infection Control, Hamilton Health \\ Sciences, Hamilton, Ontario, Canada and ${ }^{5}$ Infection Control, St. Joseph's Healthcare Hamilton, Hamilton, Ontario, Canada
}

For coronavirus disease 2019 (COVID-19), frequently reported symptoms in nonseverely sick patients include fever, fatigue, and dry cough. ${ }^{1}$ However, infected patients may not exhibit symptoms. Some patients may be presymptomatic and develop symptoms later in the disease course whereas others remain asymptomatic, but either group can be infectious. ${ }^{2,3}$

Hence, asymptomatic carriers and presymptomatic individuals may be potential sources of nosocomial transmission. As such, consideration can be given to testing asymptomatic patients upon admission to the hospital. The Infectious Diseases Society of America (IDSA) guidelines on the diagnosis of COVID-19 recommend against testing of asymptomatic hospitalized patients in lowprevalence $(<2 \%)$ settings. ${ }^{4}$ This recommendation is based on expert opinion and lacks supporting evidence.

The city of Hamilton, Ontario, Canada, has a population of 580,000 and qualified as a low-prevalence area at the time of this study. The average number of daily new cases identified was 1.9 per 100,000 population. ${ }^{5}$ For hospital admission, the testing strategy was (and continues to be) based on symptoms or exposures. ${ }^{6}$ Within this low-prevalence setting, we conducted a multicenter point-prevalence study to evaluate the utility of severe acute respiratory coronavirus virus 2 (SARS-CoV-2) testing of asymptomatic patients in terms of capturing positive cases that would be missed by symptom-based testing on admission.

\section{Methods}

We conducted a point-prevalence study across 4 tertiary acute-care hospitals in Hamilton from April 15 to April 21, 2020. The Hamilton Integrated Research Ethics Board approved this study (no. 10894).

\section{COVID-19 testing on admission}

According to provincial guidelines, testing was based on the following symptoms: fever, new or worsening acute respiratory illness symptom (ie, cough, dyspnea, sore throat, runny nose or sneezing, nasal congestion, hoarse voice, difficulty swallowing, new

Author for correspondence: Anthony D. Bai, E-mail: anthony.bai@medportal.ca Cite this article: Bai AD, et al. (2020). Utility of asymptomatic inpatient testing for COVID-19 in a low-prevalence setting: A multicenter point-prevalence study. Infection Control \& Hospital Epidemiology, 41: 1233-1235, https://doi.org/10.1017/ice.2020.349 olfactory or taste disorder(s), nausea or vomiting, diarrhea, abdominal pain), or clinical or radiological evidence of pneumonia. ${ }^{6}$ Atypical presentations included unexplained fatigue or malaise, delirium, falls, acute functional decline, exacerbation of chronic conditions, chills, headache, croup, tachycardia, decrease in blood pressure, hypoxia, and lethargy. ${ }^{6}$ At the time of this study, a patient with any of the above symptoms or exposure underwent nasopharyngeal swab testing for SARS-CoV-2 upon admission to the hospital. ${ }^{6}$

\section{Patient inclusion}

On the point-prevalence testing date, all adult inpatients were tested once if they were admitted for 7-14 days, regardless of symptoms or prior negative SARS-CoV-2 test result. Patients with a known positive SARS-CoV-2 test were excluded.

Testing on days 7-14 was based on the estimated median incubation period of 4 days (interquartile range, $2-7$ days). ${ }^{7}$ Testing after the median incubation period would have captured most COVID-19 cases, even if the exposure occurred as late as the day of admission.

\section{Testing procedure}

The nasopharyngeal swabs were collected, and a polymerase-chain reaction assay for the SARS-CoV-2 envelope and 5'-untranslated region genes was performed at the local virology laboratory in the hospital. This assay was validated against the provincial standard testing.

\section{Data collection}

Data were extracted from the patient electronic chart system, which included demographics, admitting diagnosis, hospital location, admitting service, reason for admission, Charlson comorbidity index, ${ }^{8}$ prior SARS-CoV-2 test result, chest imaging, and other microbiology test results. On the day of testing, patients were assessed for symptoms, as listed above. ${ }^{6}$

\section{Results}

Across the 4 hospitals, 125 inpatients were tested for SARS-CoV-2 (Table 1). Also, 5 patients (4.0\%) had fever and 3 patients (2.4\%) had respiratory symptoms at the time of their test. 